\title{
Dynamic Routing Implementation Decision between OSPFv3 and IS-IS in IPv6 Networks
}

\author{
Gideon Evans Norvor \\ KNUST, Kumasi, Ghana
}

\author{
Michael Asante \\ KNUST, Kumasi, Ghana
}

\author{
Anokye Acheampong \\ Amponsah \\ KNUST, Kumasi, Ghana
}

\begin{abstract}
The choice of a suitable routing protocol for implementation is an important part of every network design. During routing protocol implementation, several decisions are considered in order to select the best protocol for deployment. These decisions are usually taken on the basis of some quantitative parameters that are used to determine which protocol will perform better than others whenever there are different routing protocols available. The routing protocol with the best performance in terms of these parameters is considered the most suitable protocol and is selected for implementation. In this paper, performance of two routing protocols (OSPFv3 and IS-IS) for IPv6 has been measured and compared by simulation using Riverbed Modeler Academic Edition. Performance evaluation is based on convergence duration, IPv6 packets dropped, throughput, link utilization and queuing delay as the main parameters. The main objective of this paper is to compare both protocols and to evaluate their performance in order to determine which of them will be the more suitable one for routing network traffic in IPv6. In order to achieve the objective of this paper, two scenarios were used: OSPFv3 scenario and IS-IS scenario. Both scenarios were simulated against the chosen parameters. Overall, simulation results have shown that IS-IS performed better than OSPFv3 on the basis of convergence time, link utilization and queuing delay. OSPFv3 on the other hand performed better than IS-IS on the basis of network throughput. On the basis of IPv6 traffic dropped, IS-IS performed better than OSPFv3 when the simulation started. However, before the simulation ended, OSPFv3 dropped the least IPv6 traffic.
\end{abstract}

\section{General Terms}

Performance, Communication networks, Routing Algorithm, Network simulation, Simulation parameters.

\section{Keywords}

Dynamic routing, OSPFv3, IS-IS, and IPv6.

\section{INTRODUCTION}

The current growth of the internet has resulted in IPv4 address space exhaustion giving IPv6 the legitimacy and inevitability that cannot be ignored. IPv6 is the next-generation Internet Protocol, with a large address space, and will be used to replace the legacy IPv4 in the near future. IPv6 was completely designed on the basis of IPv4. However, some existing features in IPv4 are replaced with newly enhanced features in IPv6, and this has changed the packet layout of IPv6 making it different from IPv4 packet layout. The difference in packet structure between the two protocols means that routing traffic in IPv6 will no longer be supported by the conventional routing protocols used in IPv4 [1]. Hence new routing protocols that are compatible with IPv6 must be used. In this paper, performance of two routing protocols supported by IPv6 has been evaluated and compared by simulation. These protocols are OSPFv3 and IS-IS. Routing protocols perform a vital job in every communication network. In an IP network, the major function of routing protocols is to forward packets received from one network node to another. Routing in a communication network refers to the transmission of data from source to destination by hopping either one hop or multiple hops [2]. Routing protocols work by providing at least two services; selecting best paths between source and destination nodes, and successfully transmitting data to a specified destination [3]. Routing protocol is a combination of messages and an algorithm that enable routers to exchange routing information. Based on routing algorithms, routing protocols are able to discover available routes, construct routing tables, take routing decisions, and exchange information with each other. The routing algorithms use different metrics based on some properties of a path which helps to determine the best route to reach a destination network [4]. When it comes to larger communication networks, dynamic routing is preferred over static routing. Both static and dynamic routing are just two ways by which routers can learn about remote networks. In static routing, each network location must be entered into the routing table by the network administrator. In dynamic routing, similar routing protocols are configured on routers to enable them discover remote networks. Both routing methods have their advantages and disadvantages. In a smaller network, updating routing tables will be easy for the network administrator. However, on a larger network, doing so will be very difficult and time consuming. Hence dynamic routing protocols must be used. Using dynamic routing protocols on larger networks saves time but it also consumes network resources. Dynamic routing protocols are also more scalable; something that enables them to automatically adapt to any change in network topology. For example when a new network is added to the existing network, dynamic routing protocols are able to discover the new network automatically. Also when there is a node or a route failure, they are able to determine alternative routes and retransmit traffic via these routes with minimal disruption. Scalability is not the same for all routing protocols. Some protocols are more scalable than others. Routing protocol scalability is essential when considering current network growth rate. Therefore when deciding on which routing protocol to implement on a network, the protocol that scales well must be considered. Routing protocols are grouped into two types. These are interior gateway protocols (IGPs) and exterior gateway protocols (EGPs). Interior gateway protocols are used to enable routers exchange routing information among themselves in the same autonomous systems (AS). An AS consists of a group of networks that are solely managed by a single organization. In an AS, information in a routing table is the same for all routers. Routing Information Protocol (RIP), Interior Gateway Routing Protocol (IGRP), Enhanced Interior 
Gateway Routing Protocol (EIGRP), Open Shortest Path First (OSPF) and Intermediate System-to-Intermediate System (IS-IS), all fall under IGP. Exterior gateway protocols on the other hand are used to enable different autonomous systems to communicate. An example of exterior gateway protocol is the border gateway protocol (BGP). Interior gateway protocols differ in routing behavior and are further classified into Distance Vector Protocols, Link State Protocols and Hybrid Protocols [5]. Distance vector protocols determine best paths to a remote network on the basis of distance. Whenever a router forwards packets to another router, it is termed as a hop. The path that has the least number of hops to reach the remote network is taken as the best path. The vector points to the direction to reach the remote network. RIP and IGRP all fall under distance vector protocols. Link state protocols operate on a different principle. They create three different tables which they use in their routing process. The first table is used to store all networks directly connected to the routers. The second table is used to store the map of the complete internetwork. The third table is the routing table which is used to store the shortest path to reach all remote networks in the entire internetwork. The main distinction between these two routing algorithms is that in distance vector routing, the entire routing table content is exchanged between routers that are directly connected to each other whereas in link state routing, routers only share routing updates which contains the state of their own links with other routers in the network. OSPF and IS-IS are typical link state protocols. Hybrid protocols combine some routing characteristics of distance vector protocols and link state protocols. An example of hybrid protocol is EIGRP [5].

IPv6 is the new internet protocol developed to replace the legacy IPv4. To support routing in IPv6, the different routing protocols used in IPv4 were modified for this purpose. These protocols include Enhanced Interior Gateway Protocol version 6 (EIGRPv6), Open Shortest Path First version 3 (OSPFv3), Routing Information Protocol next-generation (RIPng) and Intermediate System-to-Intermediate System (IS-IS) for IPv6. These protocols have their advantages and disadvantages. For example research has always revealed that EIGRP converges faster than the rest of these protocols. However, it is Cisco proprietary making it hardware (router) dependent. Acquiring only one set of hardware for an internetwork will certainly come with a cost. RIPng is the successor of RIP used in IPv4. Like OSPF and IS-IS, RIP is an open standard protocol but a typical distance vector protocol [4]. Distance vector protocols do not scale well in larger networks as do by link state protocols. Therefore the choice is now left between OSPFv3 and IS-IS.

In this paper, performance of OSPFv3 and IS-IS has been evaluated and compared using Riverbed Modeler Academic Edition. Performance evaluation is based on convergence duration, IPv6 packets dropped, throughput, link utilization and queuing delay as the main parameters. Both protocols use the same routing algorithm for optimal route selection within networks but have different routing characteristics. Hence understanding their routing behavior is very important in selecting which is the more appropriate to route traffic in IPv6 networks. The main aim of this paper is to compare OSPFv3 and IS-IS and to evaluate their performance in order to determine which protocol is the more suitable one for routing network traffics in IPv6.

The objectives of the paper are: To implement the selected protocols in IPv6 network, to simulate, compare and analyze protocol performance in the same network model based on some quantitative parameters, and to recommend which of the two routing protocols is more suitable to route traffic in IPv6 network. With respect to the research objectives outlined, this paper seeks to find out which protocol will converge faster for the designed network model, which protocol will drop the least IPv6 traffic, record the highest throughput, minimum link utilization and minimum queuing delay values.

\section{RELATED WORK}

As far as routing of different network applications are concerned, volumes of simulation experiments have been performed to investigate the routing behavior of different routing protocols with much of the studies being centered on OSPF, IS-IS, and EIGRP due to their scalability over other routing protocols. Oftentimes, simulation results show that EIGRP performs better than both OSPF and IS-IS. However, EIGRP is a proprietary protocol and does not support multivendor deployment. The choice is now left between OSPF and IS-IS because they are open standard protocols. Also, a survey of related works indicated that only little is done to compare these protocols in IPv6 even as the internet gradually transit towards the new generation internet protocol. These studies are recalled as follows: [6] have performed a simulation based comparative study for OSPF, IS-IS, EIGRP and the combinations of EIGRP_IS-IS and OSPF_IS-IS using OPNET simulator. In their study, throughput, database, http object and email download response times were the parameters used to measure the performance of these protocols and their combinations. In all their five scenarios, simulation results show that the performance of the EIGRP_IS-IS protocol combination is better than the rest. [4] have carried out a simulation based performance analysis of IS-IS, OSPFv3, and a combination of both protocols for IPv6 using OPNET. Their work consists of three scenarios on which IS-IS, OSPFv3 and the combination of both protocols were configured respectively. End to end delay and variation in delay were the parameters used to measure the performance of the protocols. The network applications that were considered are voice and video. Results obtained from their simulation shows that IS-IS performs better than OSPFv3 and the combination of both protocols for video end to end delay. For variation in delay or jitter, OSPFv3 performs better than IS-IS and the combination of both protocols. For voice end to end delay, the IS-IS_OSPF combination performs better than the two. Again, [4] have carried out a simulation based performance analysis of IS-IS, OSPFv3, and a combination of both protocols for IPv6 using OPNET. Performance comparison of these protocols and their combination was carried out based on email download/upload and http page response times. Results obtained from their simulation show that IS-IS performs better than OSPFv3 and the combination of both protocols for email and http page download response times. For email upload response time, OSPFv3 performs better than IS-IS and the combination of both protocols. In [7] the performance of the combination of OSPF_IS-IS and EIGRP_OSPF_IS-IS was measured for voice and video conferencing traffic using OPNET. Performance comparison of these protocol combinations was carried out based on convergence duration, jitter, end to end delay and throughput. Results obtained from their simulation indicated that while the OSPF_IS-IS combination recorded the minimum convergence duration, the EIGRP_OSPF_IS-IS combination has shown better performance on the basis of jitter, end to end delay and throughput for both applications. In [8], Thorenoor has performed a comparative analysis on OSPF and IS-IS 
using OPNET. The main aim of her simulation experiment is to provide implementation criteria that should be considered when the choice is between OSPF and IS-IS. She divided this work into two scenarios configured with OSPF and IS-IS respectively. To measure the performance of both routing protocols, router convergence time, bandwidth utilization, throughput and queuing delay were the parameters used. Results obtained from her simulation have shown that the performance of the IS-IS network outweighed the OSPF network in terms of all the simulation parameters used. This paper contributes to ongoing research on the routing behavior of OSPF and IS-IS by comparing both protocols on the basis of convergence duration, IPv6 packets dropped, throughput, link utilization and queuing delay as the main parameters. Both routing protocols have some similarities but differ in routing behavior. They both use the same routing algorithm to determine the shortest paths to all destinations within a network.

\subsection{OSPFv3}

Open shortest path first version 3 (OSPFv3) is the modified version of OSPF that is used to support routing in IPv6. In OSPFv3, some basic techniques used in OSPF are still maintained. These techniques include designated router election, flooding, shortest path first calculation, and area support. OSPF was developed by the IETF in 1987. The version now used in IPv4 is OSPFv2. It was published in RFC 2328. OSPFv2 was later updated to OSPFv3 to support IPv6. OSPFv3 was release in 1999 and was published in RFC 5340. OSPFv3 is a link state protocol which works by using Dijkstra's algorithm to determine the shortest path to a destination within a network. To determine the shortest path to each destination, OSPFv3 first constructs a shortest path tree from the network. The shortest path tree contains all paths leading to remote networks. From the shortest path tree, OSPFv3 then selects all resulting best paths and use them to populate its routing table [5].

\subsection{IS-IS}

Intermediate System to Intermediate System (IS-IS) is an extensible intra domain routing protocol designed by Digital Equipment Corporation (DEC) as part of DECnet Phase V networks. IS-IS was made a standard routing protocol by the ISO in 1992 for communication between network devices referred to as intermediate systems [4]. The purpose of standardizing IS-IS is to make it possible for packets to be routed in the OSI protocol suite that uses the connectionless network service (CLNS) and the connectionless network protocol (CLNP) to provide a connectionless data delivery for the transport layer within the protocol stack. In order to allow the CLNS to carry IP information, IS-IS was later extended to support routing of data packets in IP, which has become the standard network layer protocol for the internet. The IP implementation of IS-IS is called integrated IS-IS. It was published in RFC 1195. The word integrated was used in the sense that the protocol can be used to support network traffic in IP environments only, OSI environments only, and can also support interconnection between hosts in both environments. In IS-IS networks, routers are called intermediate systems (ISs) and other devices are called end systems (ESs). The end systems and the intermediate systems are grouped together to form a routing domain. IS-IS also uses Dijkstra's algorithm to determine the shortest path to a destination in a network. Each IS-IS router separately builds a topology database of the network using link-state information collected from other routers in the network. Every router in the routing domain sends an IS-IS Protocol Data Unit (PDU) or a packet called Link State Packet (LSP), which contains information about itself and the links attached to it. The LSP contains information encoded in a variable length data structure that is made up of type, length, and value. This data structure is often referred to as TLV [9]. TLVs are the extensible parameter portions of the IS-IS PDUs that are used to carry different kinds of information.

\subsection{Similarities and differences between OSPFv3 and IS-IS}

- OSPFv3 and IS-IS both use Djikstra's algorithm to determine the shortest path to destination within a network.

- In a multi-access network, OSPF uses a single router called designated router (DR) to control how LSAs are flooded. The purpose of using the DR is to minimize the number of adjacencies formed so that all topology tables on routers can be synchronized. If the DR fails, OSPF uses backup designated router (BDR) for the same purpose. Election of the DR and the BDR is won by the router with the highest priority [10]. If the routers have the same router priority, router ID is used as the tiebreaker. Similar to DR, IS-IS uses designated intermediate system (DIS) for the same purpose in a multi-access network. However, there is a slight difference between the two. In OSPF, once the DR and BDR are chosen, all the other routers establish adjacency relationship with the DR and the BDR so that when the DR fails, the BDR will become the DR. In ISIS, all the routers in the broadcast medium form adjacent relationships with other routers and the DIS. When the DIS fail, any router can take over as the new DIS. Election of a DIS is based on router priority. Thus a router with the highest priority is elected as the DIS. If all the routers have the same priority, MAC address is used as the tiebreaker [11].

- Similar to OSPF, IS-IS also supports hierarchical networking allowing a larger network to be separated into logical divisions called areas. However, there is a difference between how areas are configured for both routing protocols. Whereas in OSPF a backbone area is configured and used for connecting other areas, IS-IS does not include a backbone area. The routers are grouped into a hierarchy called levels and are used to manage communication between areas. The levels are simply routers that are configured to manage communication within areas and between areas. There are two router levels defined in the hierarchy. These are level 1 and level. 2 [12]. Level 1 (L1) routers are the same as internal routers used in OSPF areas. They all have their interfaces connected within the same area. All L1 routers exchange routing information belonging to a specific area. On the other hand, Level 2 routers are used to connect different areas. They are similar to ABRs used in OSPF. An L2 router is not required to identify the topology within level 1 area but there is a possibility that an $\mathrm{L} 2$ router can be an $\mathrm{L} 1$ router in a single area [13]. 


\section{METHODOLODY}

\subsection{Simulation tool}

In this paper, Riverbed Modeler Academic Edition 17.5 is the main simulation tool used. This simulator is a GUI based and an object-oriented simulator enabling users to model real world systems in form of graphics [14]. Modeling in riverbed modeler is done on project basis. A project contains at least one scenario in which there are network devices and channels, configuration utilities, and different network application traffics that can be put together for any simulation design. The nodes and links included in the simulation represent real world network devices that are used as an input for performing the simulation.

\subsection{Simulation Design}

In this paper, two routing protocols have been compared in IPv6 network. These protocols are OSPFv3 and IS-IS. In other to achieve the objectives of the paper, the simulation was divided into two scenarios. The first scenario is an IPv6 network model configured with OSPFv3. The second scenario is a copy of the first scenario but configured with IS-IS. These scenarios were simulated on the basis of the following quantitative parameters: convergence duration, IPv6 packets dropped, throughput, link utilization and queuing delay.

\subsection{Network Topology and Connections}

Figure 3.1 shows the network topology used for the simulation. The topology models an IPv6 enterprise network consisting of four subnets. Each subnet represents a department in the company. These departments are Administration, Sales \& Marketing, Finance \& Accounting, and Information Technology.

The network topology consists of routers, network switches, firewalls, workstations, servers and an IPv32 cloud that are connected together. There are two routers, one network switch, one firewall, and 10 workstations connected together in each subnet. The routers and the firewalls in each subnet are connected together using PPP DS1 duplex link. The workstations are connected to the switch using 100BaseT duplex link. The connection between the subnets is done using PPP DS1 duplex link. In order to provide internet connection to all the subnets, an IPv32 cloud device was used. In the IT department subnet, the number of network devices connected together is the same as the other subnets. However, it has five servers connected to the switch to support some network applications. These servers are database server, remote login server, file server, http server, and email server. These servers are connected to the switch using 100BaseT duplex link.

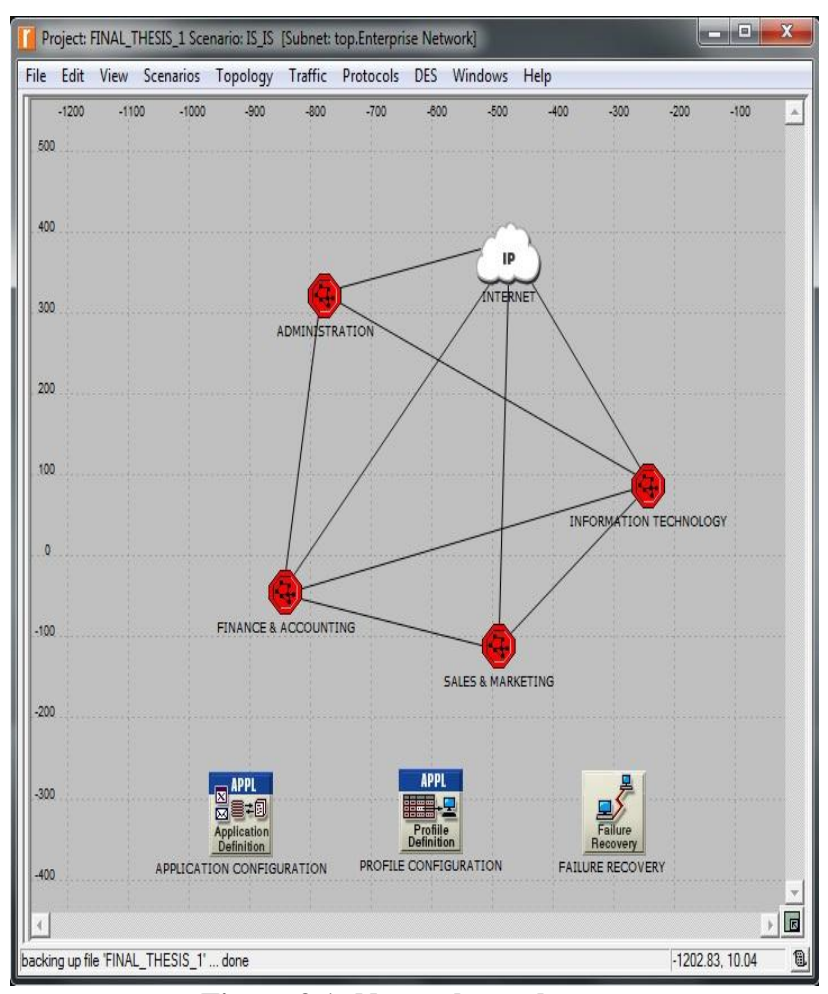

Figure 3.1: Network topology

\subsection{Application configuration}

In order to specify the selected applications and to generate network traffic for each of them in the network topology, the Application Definition and the Profile Definition objects are added from the object pallet into the modeler's workspace. Both objects are respectively renamed as application configuration and profile configuration in the modeler's workspace as shown in Figure 3.1. The application configuration object is set to support database (high load), remote login (high load), and ftp (high load). In order to generate network traffic for each application specified in the network, five profiles were defined in the profile configuration utility to support each application specified in the application configuration object.

\subsection{Node configuration}

In order to fully model the real world enterprise network, each server in the IT department is configured to support the application it is meant for. Figure 3.2 shows this configuration for the database server. 
(DATABASE SERVER) Attributes

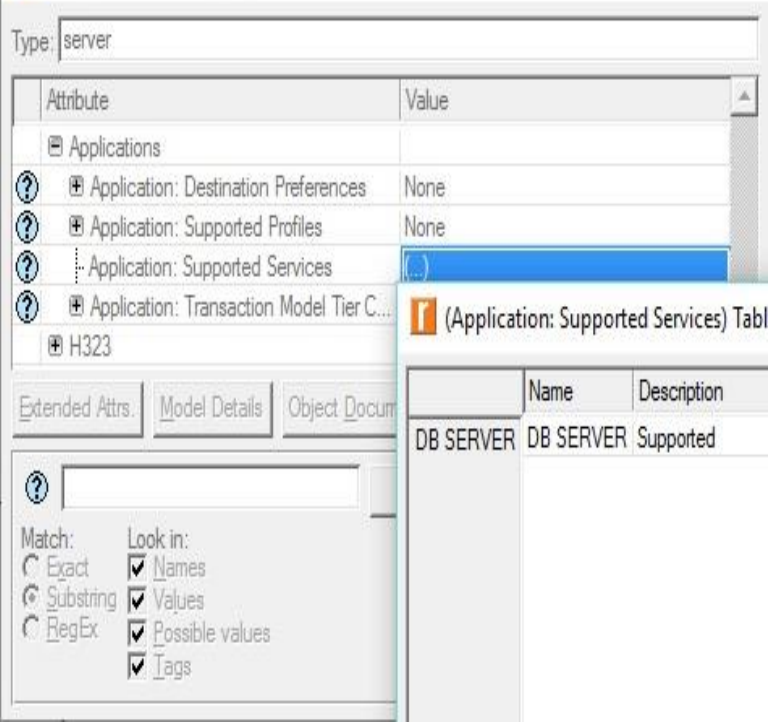

Figure 3.2: Database Server Application Configuration

Similarly, each workstation in the network topology is set to support all the applications supported in each server. Figure 3.3 shows this configuration.

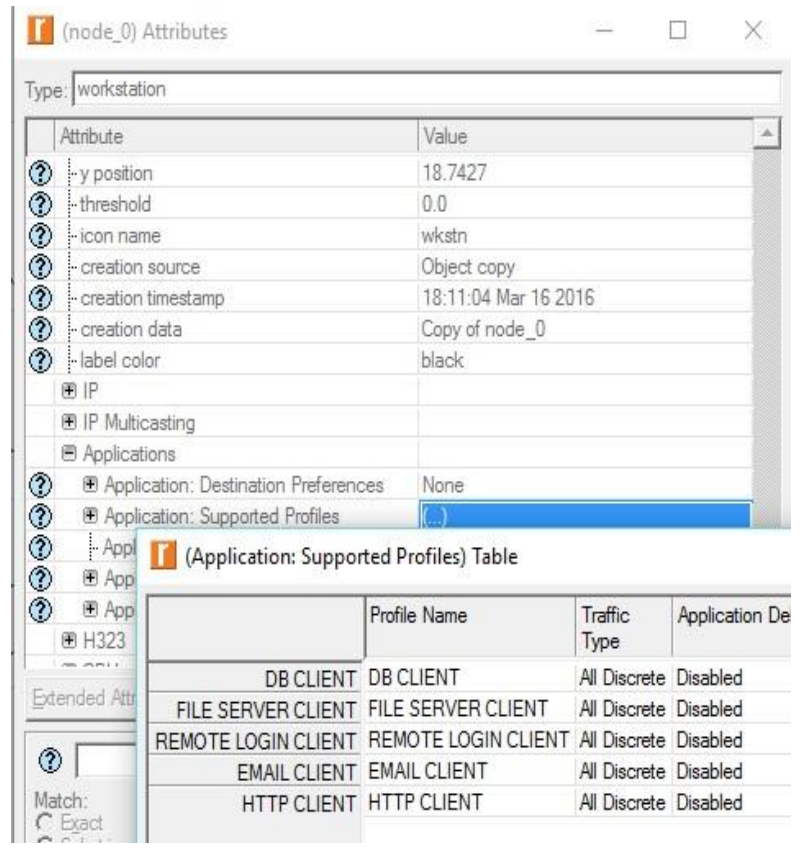

Figure 3.3: Workstation Application Configuration

\subsection{Failure Recovery Scenario}

In addition to the applications and the nodes configured, failure recovery has also been enabled in the network topology. The purpose of this is to cause some links to fail and then recover so that the network convergence duration and throughput can be measured for both scenarios. In order to achieve this purpose, the link between Sales \& Marketing department and Finance \& Accounting department is set to fail at 240 seconds and recover at 480 seconds. Figure 3.4 shows how the failure recovery is configured.

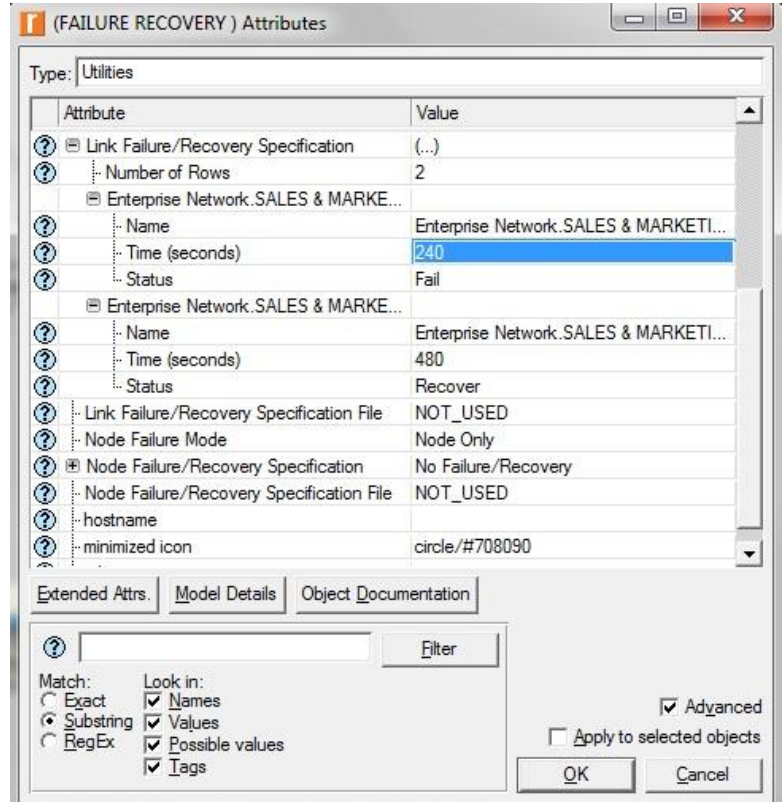

Figure 3.4: Failure Recovery Configuration

\subsection{OSPFv3 Scenario}

Figure 3.5 shows the OSPFv3 scenario used in this paper. The network topology shown in this figure is the same as the network topology described in Figure 3.1. However, in this topology, only OSPFv3 is enabled. The reason for doing this is to separately measure the effect of OSPFv 3 performance on the selected applications that are defined in the network topology. Since OSPFv3 is an IPv6 supported routing protocol, IPv6 addresses were automatically enabled in the topology before OSPFv3 was configured.

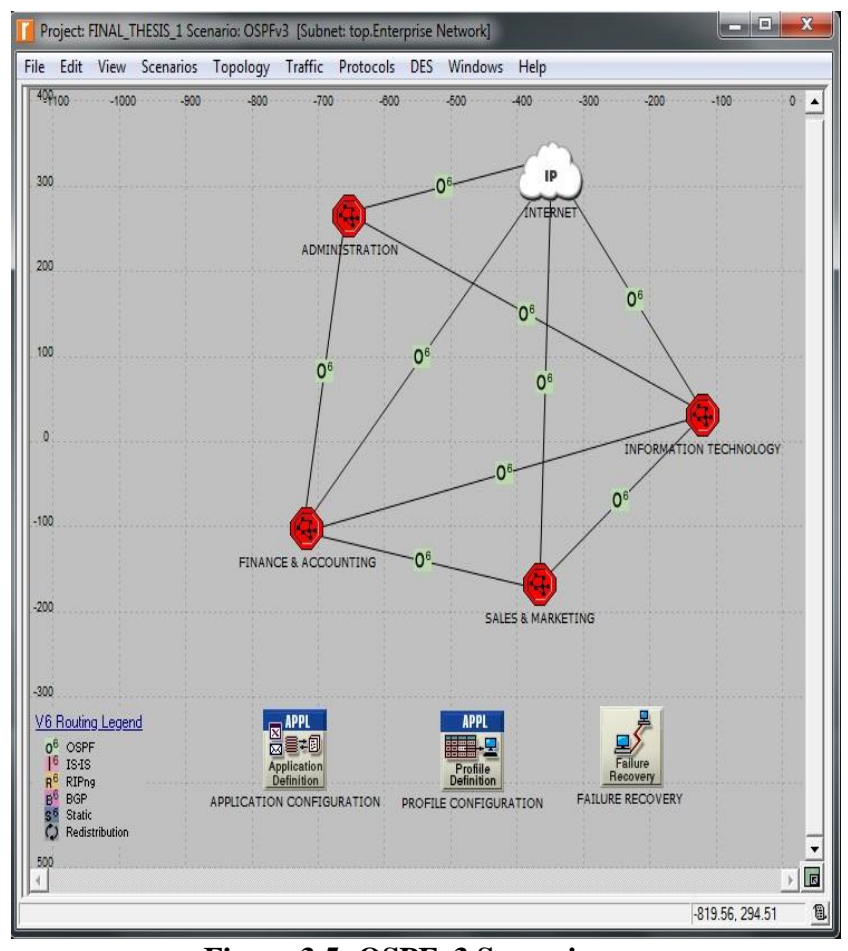

Figure 3.5: OSPFv3 Scenario

After enabling IPv6 Addresses and OSPFv3, the following parameters were chosen to measure how OSPFv3 will perform when it is used separately to route traffic in IPv6: 
convergence duration, IPv6 packets dropped, throughput, link utilization and queuing delay. After choosing these parameters, total simulation time for this scenario was set to last for 30 minutes and then the simulation was run. After the simulation, results obtained for each parameter were observed and recorded at a particular time during the simulation.

\subsection{IS-IS scenario}

Figure 3.6 shows the IS-IS scenario used in the simulation. This scenario is a copy of the OSPFv3 scenario but configured with IS-IS only. The reason for doing this is to separately measure the effect of IS-IS performance on the selected applications that are defined in the network topology. Since the performance of IS-IS is measured in IPv6, IPv6 addresses were automatically enabled in the topology before this protocol was configured.

After enabling IPv6 Addresses and IS-IS, the same parameters chosen for the OSPFv3 scenario were again chosen to simulate this scenario. This was done so that the performance of the IS-IS routing protocol can be observed and recorded. Choosing the parameters was done by following the same procedure used to set parameters in the OSPFv3 scenario.

After choosing these parameters, total simulation time for this scenario was also set to last for 30 minutes and then the simulation was run.

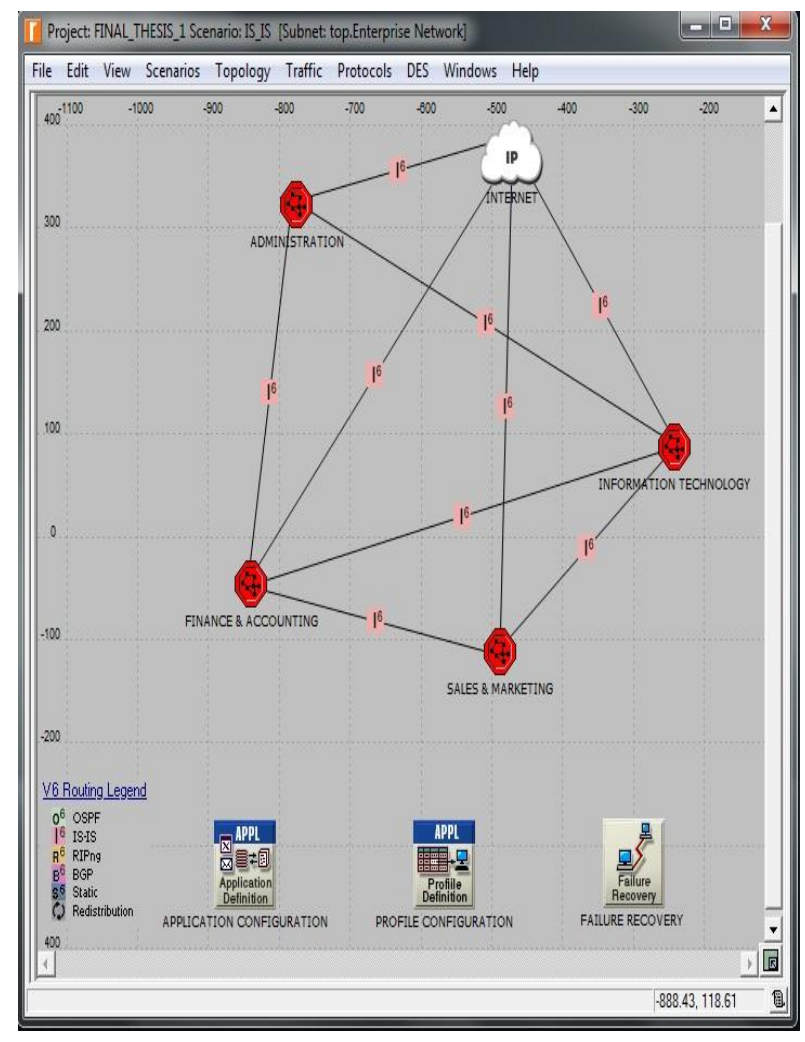

Figure 3.6: IS-IS Scenario

After the simulation, results obtained for each parameter were observed and recorded at a particular time during the simulation. These results and their corresponding simulation times are numerically shown in the following tables:

Table 3.1 shows the convergence time values recorded for both routing protocols. The respective simulation time intervals used to measure these values are shown in the table.
Table 3.1: Convergence time

\begin{tabular}{ccc}
\hline $\begin{array}{c}\text { Simulation time } \\
\text { (minutes) }\end{array}$ & $\begin{array}{c}\text { OSPFv3 } \\
\text { (seconds) }\end{array}$ & IS-IS (seconds) \\
\hline 0.5 & 12.5 & 12.3 \\
2 & 11.0 & 10.9 \\
3 & 10.0 & 9.9 \\
4 & 9.0 & 8.9 \\
5 & 7.0 & 6.9 \\
8 & 2.4 & 2.3 \\
\hline
\end{tabular}

Table 3.2 shows the throughput values recorded for both OSPFv3 and IS-IS after the simulation. The throughput values measured and their respective simulation times are shown in the table.

Table 3.2: Throughput

\begin{tabular}{ccc}
\hline $\begin{array}{c}\text { Simulation time } \\
\text { (minutes) }\end{array}$ & OSPFv3 (bits/sec) & IS-IS (bits/sec) \\
\hline 0.5 & 1,590 & 425 \\
2 & 600 & 200 \\
4 & 380 & 140 \\
5 & 300 & 100 \\
10 & 190 & 50 \\
15 & 170 & 40 \\
20 & 100 & 30 \\
\hline
\end{tabular}

Table 3.3 shows how the Sales \& Marketing and Finance \& Accounting link was utilized by OSPFv3 and IS-IS. The link utilization values measured and their respective simulation times are shown in the table.

Table 3.3: Link utilization

\begin{tabular}{ccc}
\hline $\begin{array}{c}\text { Simulation time } \\
\text { (minutes) }\end{array}$ & OSPFv3 (\%) & IS-IS (\%) \\
\hline $0-1$ & $0.060-0.102$ & $0.010-0.028$ \\
5 & 0.018 & 0.008 \\
10 & 0.010 & 0.005 \\
15 & 0.008 & 0.004 \\
20 & 0.005 & 0.002 \\
25 & 0.004 & 0.002 \\
30 & 0.003 & 0.002 \\
\hline
\end{tabular}

Table 3.4 shows the queuing delay values measured in the Sales \& Marketing and Finance \& Accounting link for both OSPFv3 and IS-IS. The respective simulation time intervals used to measure these values are shown in the table.

\begin{tabular}{ccc}
\multicolumn{3}{c}{ Table 3.4 Queuing delay } \\
\hline $\begin{array}{c}\text { Simulation time } \\
\text { (minutes) }\end{array}$ & OSPFv3 (seconds) & IS-IS (seconds) \\
\hline 0 & 0.00074 & 0.00050 \\
0.5 & 0.00070 & 0.00054 \\
5 & 0.00048 & 0.00043 \\
10 & 0.00048 & 0.00044 \\
15 & 0.00049 & 0.00044 \\
20 & 0.00049 & 0.00045 \\
25 & 0.00050 & 0.00046 \\
\hline
\end{tabular}


Table 3.5 shows the values recorded for IPv6 traffics dropped in the OSPFv3 and IS-IS networks. The IPv6 traffics dropped values measured and their respective simulation times are shown in the table.

Table 3.5: IPv6 Traffics Dropped

\begin{tabular}{ccc}
\hline $\begin{array}{c}\text { Simulation time } \\
\text { (minutes) }\end{array}$ & $\begin{array}{c}\text { OSPFv3 } \\
\text { (packets/sec) }\end{array}$ & $\begin{array}{c}\text { IS-IS } \\
\text { (packets/sec) }\end{array}$ \\
\hline 2.5 & 4.25 & 4.25 \\
5 & 4.25 & 3.40 \\
10 & 1.6 & 2.98 \\
15 & 1.1 & 2.90 \\
20 & 0.8 & 2.80 \\
25 & 0.7 & 2.70 \\
30 & 0.6 & 2.60 \\
\hline
\end{tabular}

\section{SIMULATION RESULTS}

This section presents the discussion of results obtained from the simulation. Each result is obtained based on the parameters chosen to measure the performance of both routing protocols. The results are presented in form of graphs. Riverbed Modeler Academic Edition 17.5, which is the main simulator used is configured to produce a graphical result of all the simulation parameters chosen.

\subsection{Convergence Time}

The convergence time of both routing protocols is shown in Figure 4.1. From this figure, it can be observed that convergence duration for both routing protocols is nearly the same. However, there is a slight variation as shown in the graph. At exactly 0.5 minute into the simulation, IS-IS converged a little faster than OSPFv3. The value for convergence time in the IS-IS network at this time is 12.3 seconds while it is 12.5 seconds in the OSPFv3 network. Both protocols took these times to converge because they need to build their neighbor, topology and routing tables in order to have a complete knowledge about the network before they can start forwarding packets. Between 2 to 3 minutes during simulation, IS-IS slightly shows better performance over OSPFv3. When the link failed at 240 seconds (4 minutes), the convergence time of IS-IS and OSPFv3 decreased to 9.0 and 8.9 seconds respectively. When the link recovered at 480 seconds ( 8 minutes), convergence duration for both protocols again decreased to around 2.4 and 2.3 seconds respectively. Convergence duration for both protocols decreased at this time because their routers only need to communicate the link failure and update their topology tables. Although convergence time for both routing protocols keeps decreasing until the simulation ended, IS-IS converged faster than OSPFv3. The reason for this routing behavior might be the minimal number of packets IS-IS uses during its routing process. When there is a route or node failure in a network, link-state protocols will have to update their topology databases. While OSPFv3 routers will have to flood LSAs to their neighbors in order to recalculate entries in their routing table, IS-IS routers will have to flood LSPs to their neighbors in order to do the same. Though the routing process remains the same for both protocols during network topology changes, OSPFv3 uses more packets and as a result it takes much time to converge. Comparing these results to those obtained by [8] confirmed that IS-IS has a better convergence time than
OSPF. In her simulation, whereas both IS-IS and OSPF initially took 5.4 and 29 seconds to converge, both routing protocols initially took 12.3 and 12.5 seconds to converge in this simulation. Even though the difference in the numerical values obtained here is small, all numerical values measured for IS-IS show that IS-IS recorded the faster convergence time.

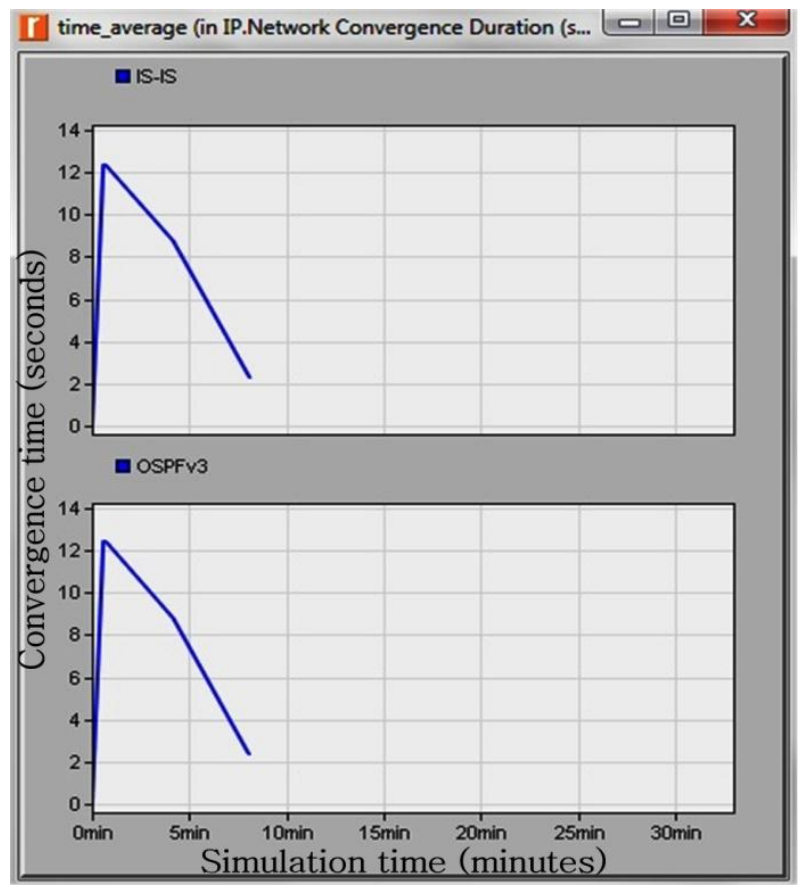

Figure 4.1: Network Convergence Duration (seconds)

\subsection{Throughput}

Network throughput is an important parameter. This parameter is used to measure the average number of bits received or transmitted successfully by the receiver or the transmitter channel per second. Measuring network throughput is done in bits per second or sometimes in packets per second [14]. The throughput obtained from the Sales \& Marketing and Finance \& Accounting link is shown in Figure 4.2. It can be seen in this figure that the OSPFv3 network has a higher throughput than the IS-IS network. At around 0.5 minute during simulation, the average number of bits transmitted successfully via the Sales \& Marketing and Finance \& Accounting link per second in the OSPFv3 network is about 1,590 bits. This value is about four times higher than the 425 bits delivered through the same link in the IS-IS network. As the link fails at 4 minutes, throughput values for both scenarios drop significantly. While the throughput value for OSPFv3 falls to 380 bits, the value for IS-IS falls to 140 bits. Even as the link recovered at 8 minutes during the simulation, protocol performance became very poor as the throughput values for both OSPFv3 and IS-IS respectively fall below 200 and 100 bits/sec. However, performance of the OSPFv3 network is better than the IS-IS network. The reason for this poor performance is as a result of the process each protocol has to go through after the link failure before it can converge again. When a link failure occurs within a network, link-state protocols take some time to converge and this consequently affects network throughput. 


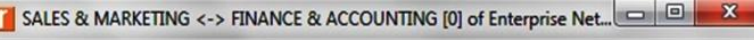

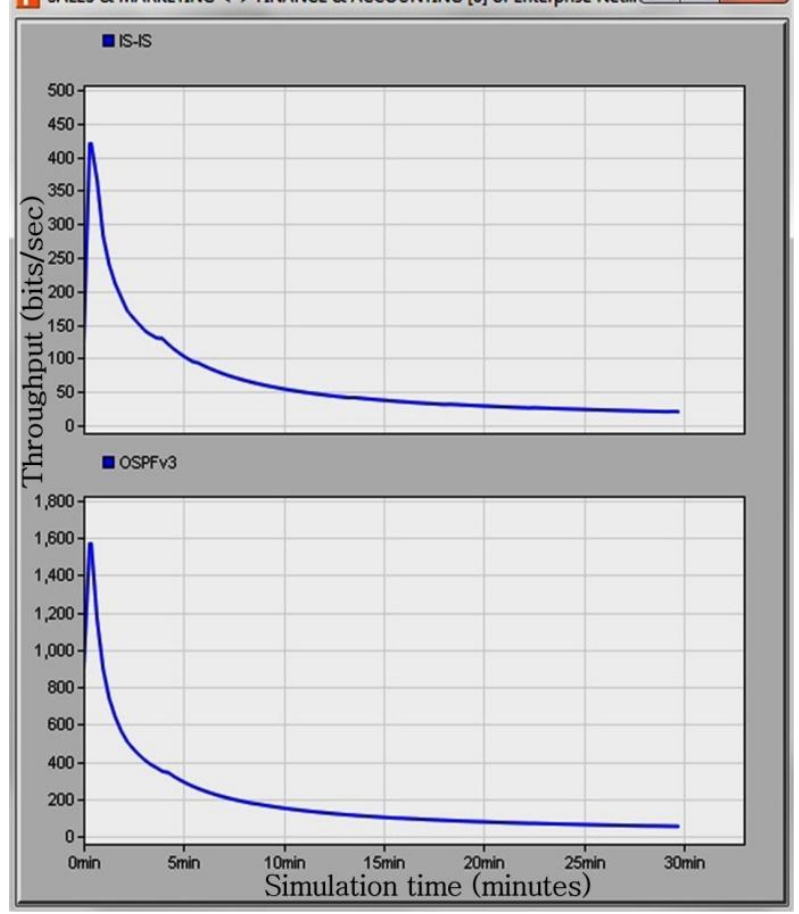

Figure 4.2: Throughput (bits/sec)

\subsection{Link utilization}

Link utilization measures how much of network bandwidth (in percent) is being consumed by traffics generated in the network. Figure 4.3 shows how the Sales \& Marketing and Finance \& Accounting link was utilized for both routing protocols. It can be observed that as the simulation starts, link utilization value for IS-IS increases from $0.01 \%$ to about $0.028 \%$. At around 3 minutes, this value decreases back to $0.01 \%$ and then continues to decrease from 5 minutes through to the end of the simulation. In the case of the OSPFv3 network, link utilization value increases from $0.06 \%$ at the start of simulation time and then increases to $0.102 \%$. At around 5 minutes, this value decreases to $0.018 \%$ and then continues to decrease until the end of simulation time. Comparing these values, it can be concluded that IS-IS has the minimum link utilization and hence it is better than OSPFv3 in terms of this parameter. This happened as a result of the small amount of network traffic received in the IS-IS network. If traffic received in the network is high it is an indication that link utilization will be high and the possibility that link congestion will happen is also high. This was the case of the OSPFv3 network.

\subsection{Queuing Delay}

This parameter measures the time taken by each packet to wait in a queue before it can be forwarded over a link. Queuing delay can be caused by several factors. For example when the speed of the link via which a router received packets is faster than the speed on the link through which the router can forward these packets, queuing delay can occur. Figure 4.4 shows results obtained for queuing delay in both scenarios used in this paper. It can be observed that as the simulation started, queuing delay value for IS-IS started from 0.00050 second and increased to 0.00054 second. This value then began to decrease gradually and finally settled at 0.00046 second when the simulation ended. In contrast to the OSPFv3 network, this is different. Even though queuing delay started from 0.00074 and began to decrease gradually during simulation, the value recorded at the end of the simulation ( 0.0005 second) is still greater than the value recorded in the IS-IS network. Hence on the basis of queuing delay, IS-IS performed better than OSPFv3.

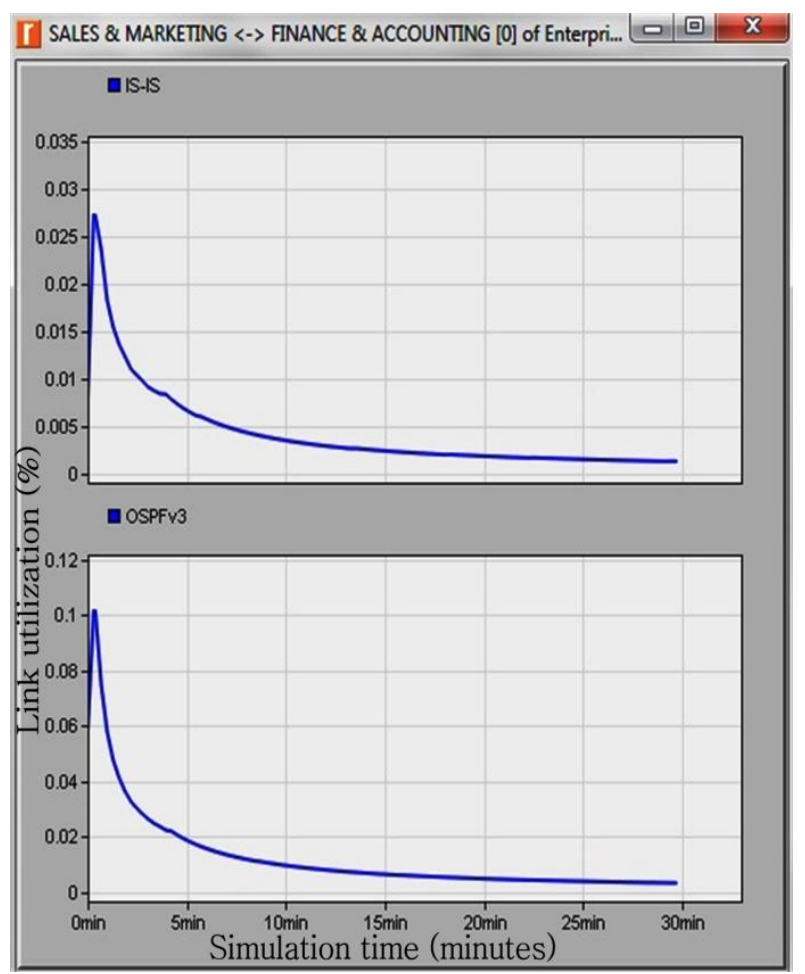

Figure 4.3: Link utilization (\%)

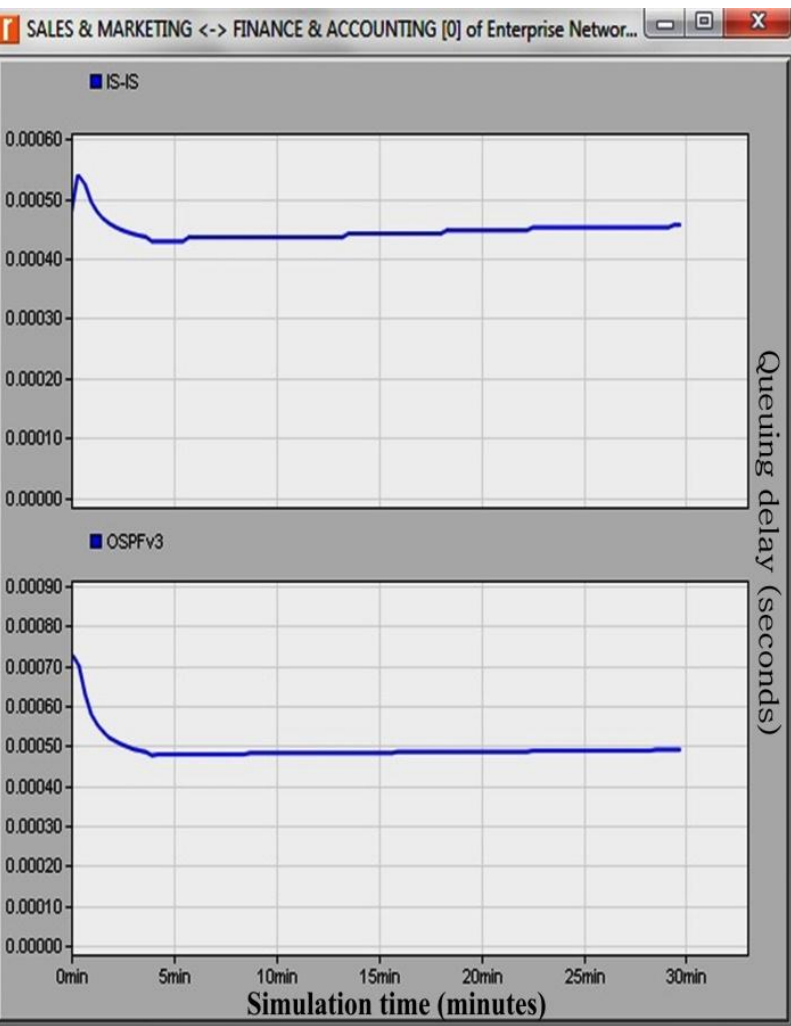

Figure 4.4: Queuing delay (seconds) 


\subsection{IPv6 Traffics Dropped}

This parameter measures the total amount of IPv6 packets lost by every node within a network. Several reasons could lead to a packet being dropped in a network. For example when more traffic is forwarded through the network, the network can become congested leading to higher bandwidth utilization. In a congested network, packet delivery is delayed. When this happens some packets are dropped without reaching their destination. Figure 4.5 shows IPv6 traffic dropped by both OSPFv3 and IS-IS. From this figure, it can be seen that both protocols dropped a maximum number of 4.25 packets per second within 2.5 minutes of simulation time. However, in the IS-IS network, this value started from 0 and then rises to the maximum value (4.25) before it begins to decrease. In the OSPFv3 network, this value rather started from 3.7 and then increased to the maximum value (4.25). OSPFv3 maintained this value through to about 4 minutes before it started decreasing. In the IS-IS scenario, the value for this statistic starts dropping from around 3 minutes during simulation time. At around 10 minutes, the number of IPv6 traffic dropped in the IS-IS network is around 2.9 packets. Just before the end of simulation time, this valued gradually reduced to about 2.6 packets per second. Even though when the simulation was about to end, total number of IPv6 traffic dropped in the OSPFv3 network significantly reduced to 0.6 packets per second. OSPFv3 dropped more IPv6 packets when the simulation stated. This must have happened as a result of the more traffic received in the OSPFv3 network indicating the possibility of network congestion.

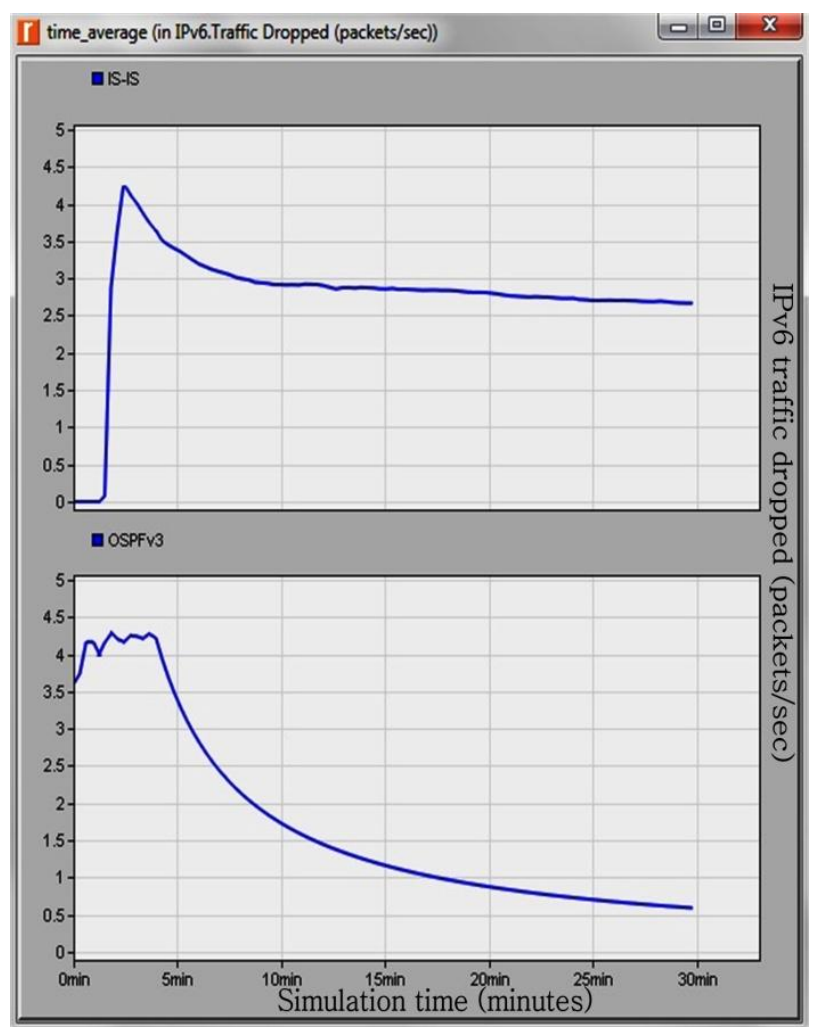

Figure 4.5: IPv6 Traffic Dropped (packets/sec)

\section{CONCLUSION}

In this paper, performance of two routing protocols (OSPFv3 and IS-IS) for IPv6 has been measured and compared by simulation. Performance evaluation was carried out on the basis of the following quantitative parameters: network convergence duration, IPv6 traffic dropped, throughput, link utilization, and queuing delay. On the basis of convergence time, simulation result obtained indicated that the convergence duration of IS-IS is a little faster than that of OSPFv3. It was observed that when there was a link failure routers in the IS-IS network learned the topology faster than the routers in the OSPFv3 network. Hence IS-IS performed better than OSPFv3 in terms of this parameter. On the basis of throughput, it is observed that the point-to-point throughput of OSPFv3 is higher than IS-IS. In terms of which protocol has recorded the minimum link utilization values, simulation results indicated that IS-IS performed better among the two routing protocols. The more traffic received in the OSPFv3 network is an indication that more bandwidth was utilized in the OSPFv3 network. On the basis of which protocol dropped the least IPv6 traffic, simulation results have indicated that IS-IS performed better than OSPFv3 in terms of this parameter when the simulation started. Because more traffic was received in the OSPFv3 network when the simulation started, this led to network congestion and as a result OSPFv3 dropped more IPv6 packets when the simulation started. However, before the end of the simulation, OSPFv3 dropped the least IPv6 traffic. Based on these simulation results, it can be concluded that overall, the performance of IS-IS outweighed that of OSPFv3.

\section{REFERENCES}

[1] Genkov, D. (2011). An approach for finding proper packet size in IPv6 networks. In Proceedings of the $12^{\text {th }}$ International Conference on Computer Systems and Technologies, 442-447. ACM.

[2] Kannagi, P., Rajasekar, M. (2013). Performance comparison of routing protocols (OSPF \& EIGRP). International Journal of Advanced Research, 1 (3) 13-22.

[3] Lemma, E. S. \& Angelo, W. (2009). Performance comparison of EIGRP/IS-IS and OSPF/IS-IS [www.diva-portal.org].

[4] Kaur, J., Singh, P. (2014). Simulation based performance analysis of IPv6 based IS-IS, OSPFv3 and OSPFv3_ISIS protocols. International Journal of Software and Hardware Research Engineering, 2 (8), 25-28.

[5] Lammle, T. (2007). CCNA: Cisco Certified Network Associate, Study Guide, 6th edition. Indiana, Indianapolis, Wiley Publishing, Inc.

[6] Pandey, N., Kumar, D., Palwal, H. (2015). Simulation based comparative study on EIGRP/IS-IS and OSPF/ISIS. International Journal of Engineering Research and General Science, 3 (2), 204-214.

[7] Farhangi, S., Rostami A., Golmohammadi, S. (2012). Performance comparison of mixed protocols based on EIGRP, IS-IS and OSPF for real-time applications. Middle-East Journal of Scientific Research, 2(11), 15021508, doi 10.5829/idosi.mejsr.2012. 12.11.144.

[8] Thorenoor, S. G. (2010). Communication service provider's choice between OSPF and IS-IS dynamic routing protocol and implementation criteria using OPNET. In second International Conference on Computer and Network Technology (ICCNT), Bangkok, 38 (42), 23-25.

[9] Hopps, C. (2008). Routing IPv6 with IS-IS. RFC5308.[https://www.rfceditor.org/rfc/rfc5308.txt], 
(accessed 2016 February 18).

[10] Cisco. (2016). Cisco Nexus 7000 Series NX-OS Unicast Routing Configuration Guide, ReleaseRelease5.x[http://www.cisco.com/c/en/us/td/docs /switches/datacenter/sw/5_x/nxos/unicast/configuration/g uide/13_cli_nxos/13_ospfv3.html],(accessed 2016 March)

[11] Empson, E. (2007). CCNP BSCI Portable Command Guide. Cisco Press.

[12] Kaur, A., Kumar, E. D. (2015): Comparative analysis of link state protocols OSPF and IS-IS International Journal of Computer Science Trends and Technology, 3 (4), $159-168$.

[13] Roussinos, P. A. (2014). Performance comparison of OSPF and IS-IS routing protocols in dual-stack enterprise networks. (Doctoral dissertation, Edinburgh Napier University), [soc.napier.ac.uk], (accessed 2016 January 4).

[14] Pan, J., \& Jain, R. (2008). A survey of network simulation tools: Current status and future developments. [www.cse.wustl.edu], (accessed 2015 December 7). 